\title{
Ex vivo analysis of renal proximal tubular cells
}

\author{
David Legouis ${ }^{1}$, Aurélien Bataille ${ }^{1}$, Alexandre Hertig ${ }^{1,2,3}$, Sophie Vandermeersch ${ }^{1}$, Noémie Simon ${ }^{1}$, \\ Eric Rondeau ${ }^{1,2,3}$ and Pierre Galichon ${ }^{1,2,3^{*}}$
}

\begin{abstract}
Background: Experimental models are inevitably a compromise between accurately reproducing a pathological situation and schematically simplifying it, which is intended to provide both relevance and conclusiveness. In-vivo models are very relevant, but multiple cell-types undergoing various changes may hinder the observation of individual molecular events.

Results: Here, we describe a method for analyzing and isolating specific cell types from the kidney and studying the phenotype they have acquired in vivo. Using flow cytometry, immunofluorescence, and RT-PCR, we show that our method is suitable for studying and isolating proximal tubular cells with an anti Prominin-1 antibody. Kidneys are subjected to mechanical dissociation followed by flow-cytometry analysis. Hundreds of thousands of proximal tubular cells are then isolated by magnetic separation followed by direct analysis or primary cell culture. Using our method, we detect phenotypic changes in the proximal tubular cells after renal ischemia reperfusion, and we isolate the proximal tubular cells, with a purity over $80 \%$.

Conclusions: This method is efficient, quick, simple, and cheap, and should be useful for studying cell-type specific parameters after in vivo experimental studies. It is also a simple method to obtain a specific primary cell culture from any animal strain.
\end{abstract}

Keywords: Organ physiology, Experimental models, Cell phenotype, Primary cell cultures, In vivo, In vitro

\section{Background}

Animal studies are performed in controlled environments, and provide an acceptable compromise for studying events that occur in human beings, providing clues for understanding when and where such events originally occur. For example, sufficient technological progress has now been achieved to make it possible to see, literally, when and where an acute kidney injury will induce patchy necrosis of tubular epithelial cells [1]. Obtaining mechanistic insight into why and how this occurs is another similarly important challenge: new drug discovery implies the ability to distinguish between the molecular causes and the biological effects in specific cell types. In-vitro studies clearly provide an important technical complement here [2-5]. The biological relevance of what is observed in a culture dish is, however, very uncertain, because the study environment is even more restricted. Furthermore, the cells are completely isolated from the

\footnotetext{
* Correspondence: galichon@orange.fr

'INSERM, UMR-S1155, Paris, France

2UPMC, Sorbonne Universités, Paris 6, France

Full list of author information is available at the end of the article
}

source organ, as well as from the whole source animal. Not to mention the fact that immortalizing a cell lineage, something routinely performed to save time and money, may profoundly alter cell programming and how it responds to injury.

$E x$-vivo study of the cell lysate of a whole organ subjected to some form of injury would obviously be uninformative, since it would mix many different cell types and could provide misleading observations or mask significant ones. Another approach would be to examine the population of interest after the injury has been imposed in vivo (for instance, looking at proximal tubular epithelial cells in the context of an acute kidney injury after trying to sort and capture living cells belonging to the population of interest ex vivo). The limitations of this approach so far have been 1) the dissociation, using enzymes at $37^{\circ} \mathrm{C}$, which can dramatically modify the cell phenotype (extracellular protein cleavage and transcriptomic changes may occur as early as 10 minutes after enzymatic exposure) [6-8], and 2) the heterogeneity of the dissociated cell suspension which, as far as we know, was always cultured 


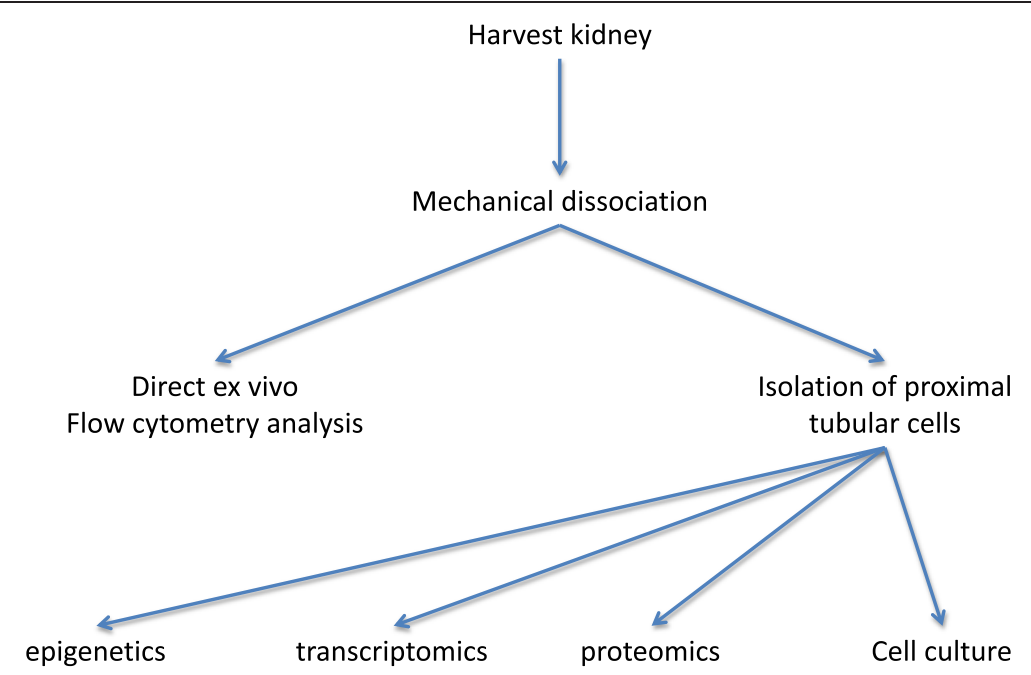

Figure 1 Flowchart of the method detailing the three applications of dissociated cells: direct analysis, isolation of a subpopulation of cells for subsequent analysis, or for primary cell culture.

before being studied (except for interstitial cells: leucocytes and fibroblasts) [9].

Here, we describe a fast, low-cost method that keeps the cells alive and does not necessitate costly equipment, that can be used to extract thousands of intact proximal tubular cells from one or a few fresh kidneys, making it possible to carry out ex vivo and extemporaneous quantification of multiple molecular pathways or cell-type specific selection for subsequent analysis or culture (Figure 1).

\section{Results and discussion}

Instantaneous, enzyme-free preparation of a cellular suspension from whole kidneys

Immediately after being harvested from adult C57bl6/J mice, each kidney was immersed in dissociating buffer, chopped and dissociated using the GentleMACS cell dissociator (Miltenyl Biotec, California, USA) for 2 minutes

Table 1 Protocol for direct dissociation of a whole kidney into a cell suspension

1. Prepare sterile dissociation buffer $\left(4^{\circ} \mathrm{C}\right)$ : PBS $1 \times, 0.5 \%$ bovine serum albumin, and 2 mM EDTA.

2. Immerse the kidney in $1 \mathrm{~mL}$ of this dissociation buffer

3. Remove the capsule and roughly chop the kidney with a surgical blade

4. Transfer the solution obtained into GentleMACS C-tubes.

5. Use the brain_03 and then the spleen_04 program of the GentleMACS dissociator.

6. Pass the solution obtained through a 30- $\mu \mathrm{m}$ sieve.

7. Rinse with $4 \mathrm{~mL}$ of dissociation buffer.

8. Centrifuge at $500 \mathrm{~g}$ for10 minutes.

9. Discard the supernatant.

10 Resuspend cell pellet in $180 \mu \mathrm{L}$ of dissociating buffer. at $4^{\circ} \mathrm{C}$, with no added enzymes. This protocol, which takes no more than 10 minutes total, is detailed in the Methods section and in Table 1.

\section{Debris exclusion prior to cell analysis}

The suspension contained cells of various sizes and structures, plus many cellular fragments and aggregates that would prevent proper gating using the classical FSC/SSC (size/structure) plot on a cytometer. It should be noted that debris resulting from mechanical dissociation of the kidney can lead to significant autofluorescence; this debris must be excluded in order to obtain a reliable and accurately quantitative signal. Because debris does not contain chromatin, Hoechst staining was used firstly to 1) detect DNA (real cells) and to exclude non-cellular debris, and 2) among intact cells, select singlets using a peak versus area plot (H/A, Additional file 1: Figure S1). Autofluorescence preponderantly emits in the green channels, which is why we used the V2 signal to compensate for the V1 signal, further excluding autofluorescent debris. This further improved the quantification of DNA cellular content, and made it possible to analyze the cell-cycle (Figure 2).

\section{Ex-vivo analysis of proximal tubular cells in the whole kidney suspension}

Proximal tubular epithelial cells are highly differentiated renal cells. Prominin-1 is a surface glycoprotein expressed in the brush border [10-12]. Anti-prominin-1 antibodies are theoretically appropriate for use in that a) they are commercially available (anti-Prominin-1 rat monoclonal IgG1 antibody, clone MB9-3G8, Miltenyi Biotec, Bergisch Gladbach, Germany), b) they have been conjugated with a number of tags, and c) they target the extracellular region 

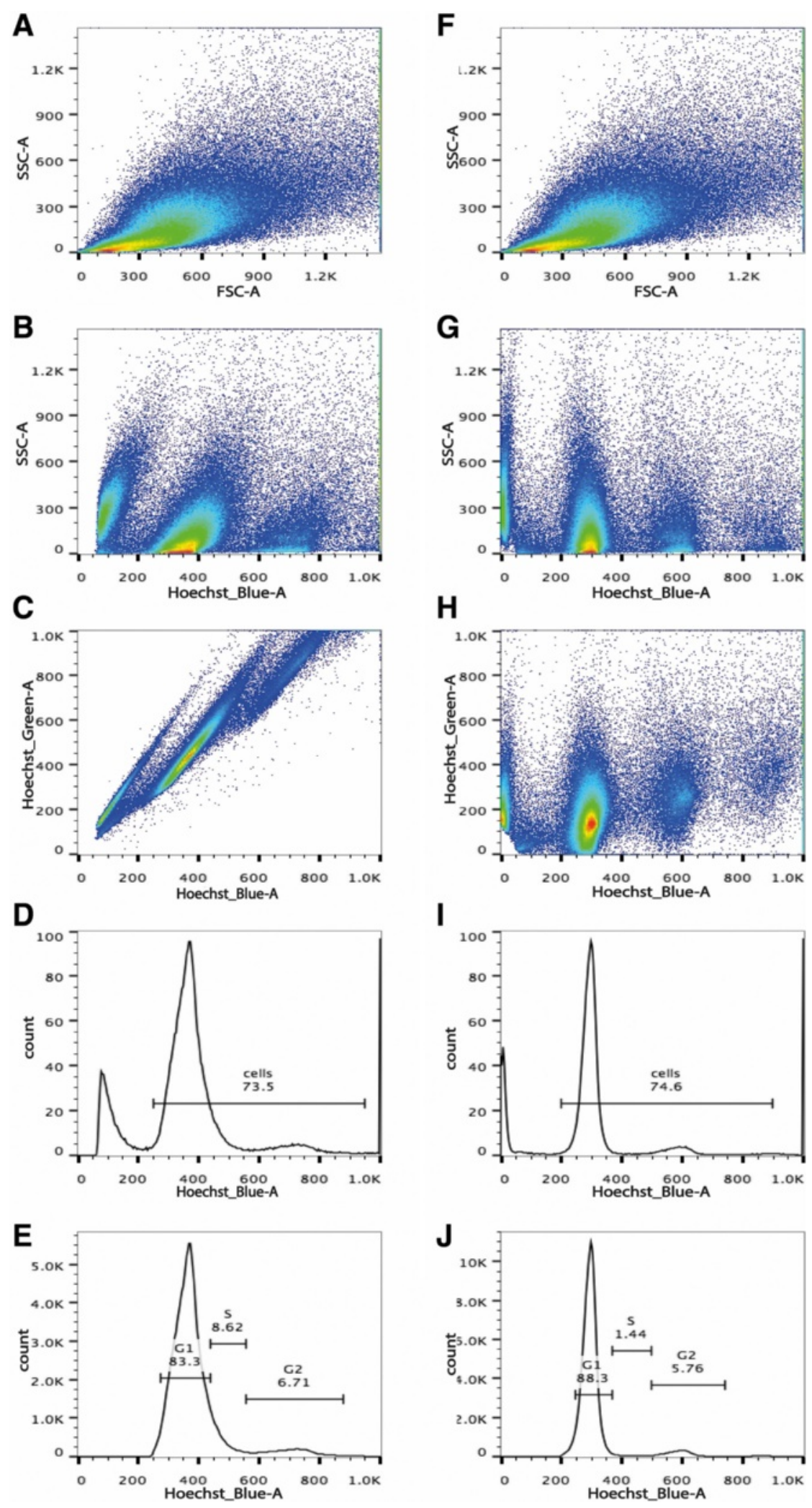

Figure 2 (See legend on next page.) 
(See figure on previous page.)

Figure $\mathbf{2}$ Gating strategy for flow cytometry analysis of dissociated cells. A \& F. Density plot displaying Side scatter versus Forward scatter before (A) and after (F) compensation for autofluorescence. B \& G. Representation of Hoechst staining in dot plots versus Side scatter before (B) or after (G) compensation for autofluorescence. $\mathbf{C} \& \mathbf{H}$ Representation of Hoechst staining in dot plots versus Hoechst Green signal before (C) or after $\mathbf{( H )}$ compensation for autofluorescence. The compensation breaks the correlation between SSC or Hoechst's green signal with Hoechst's blue signal. D \& I. Representation of the cell cycle before (D) and after (I) compensating for autofluorescence. E \& $\mathbf{J}$. The first peak corresponds to G1 cycle cells, whereas the second peak to the G2 cycle cells, with cells in phase S in between. Compared to non- compensated sample (E), compensation (J) enhances the precision of the cell cycle study.

of prominin-1, which makes it possible to work with live, unpermeabilized cells.

There are several isoforms of Prominin-1, and some antiProminin-1 antibodies show clone-dependent specificities for renal epithelial cellular subtypes [13]. To confirm that Prominin $-1^{+}$cells detected with clone MB9-3G8 were indeed proximal epithelial tubular cells, we measured its coexpression with megalin, a reliable marker of proximal tubules, by means of two different techniques. Using immunofluorescence and flow cytometry, we showed that Prominin-1 and Megalin colocalize (Figure 3 and Additional file 2: Figure S2 and Additional file 3: Figure S3). Megalin and Prominin-1 also colocalized after ischemia reperfusion injury (Figure 3 and Additional file 4: Figure S4).

We studied the effect of ischemia reperfusion injury on the expression of prominin-1 and CD44 (a marker expressed by injured renal epithelial cells) in cells from dissociated kidneys. In control mice, Prominin-1 was positive in 34.2 and $31.1 \%$ of the cells, and CD44 was expressed respectively in $6.42 \%$ and $9.04 \%$ of these Prominin $-1^{+}$cells. At day 2 post ischemia reperfusion injury, Prominin-1 was positive in 51.7 and $48.9 \%$ of the cells, and CD44 was expressed respectively in $33.3 \%$ and $21.9 \%$ of these Prominin- $1^{+}$cells (Figure 4 ).

\section{Isolation of proximal tubular cells from a whole kidney cell suspension}

We reasoned that we could capture proximal tubular cells by using magnetic microbeads conjugated with an antiprominin-1 antibody that specifically targets the proximal tubules; thus, a strong magnetic field applied to the preparation would separate the proximal tubular cells from the others. We therefore incubated the kidney cell suspension obtained as described above with microbead-conjugated anti-prominin-1 antibodies. Allophycocyanin (APC)-conjugated anti-prominin-1 antibodies were also added to label Prominin $-1^{+}$cells in order to check the quality of the magnetic separation by flow cytometry. We chose APC

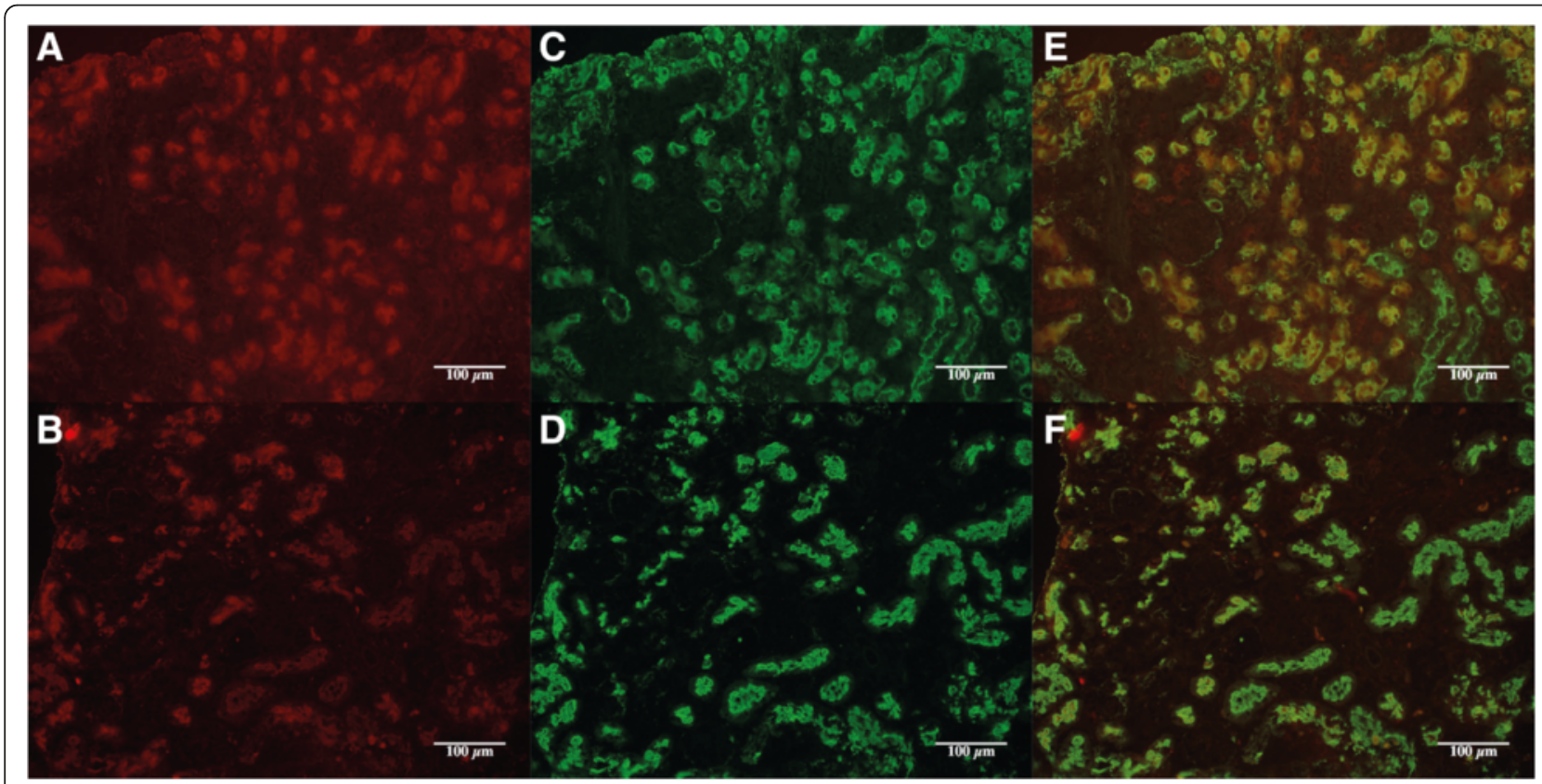

Figure 3 Immunofluorescence analysis of control (A, C, E) and fibrotic kidneys (B, D, F) at magnification $\mathbf{x} 20$. Prominin-1 (red) staining is shown in $\mathbf{A}$ and $\mathbf{B}$, Megalin (green) staining is shown in $\mathbf{C}$ and $\mathbf{D}$, and double staining is shown in $\mathbf{E}$ and $\mathbf{F}$. Expression of megalin and prominin1 colocalize on the brush border of renal proximal tubular epithelium in mice. In fibrotic kidneys, tubules are interspaced with fibrosis but still express Prominin-1 and Megalin. 


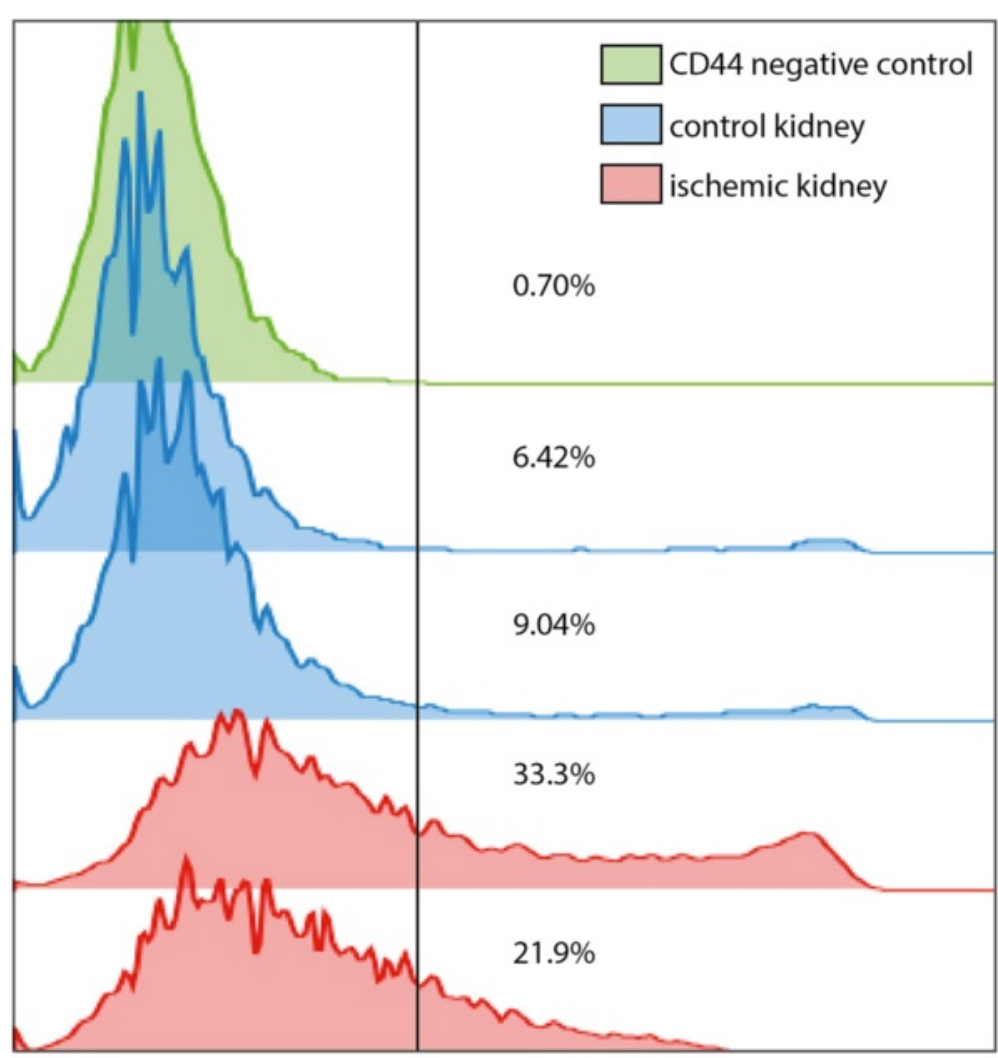

\section{CD44 expression in Prominin-1+ cells}

Figure 4 Expression of CD44 by prominin-1 $1^{+}$cells in a suspension of dissociated cells from control and ischemia reperfusion injured kidneys. In green, cells are stained for prominin-1 but not for CD44 (negative control, $0.7 \%$ false positivity for CD44). In Blue, cells from control kidneys stained for Prominin-1 and CD44 (rare CD44+ cells). In red, cells from ischemia-reperfusion injury kidneys stained for Prominin-1 and CD44 (increased number of CD44+ cells).

since its emission wavelenghth is barely affected by autofluorescence [14], and because it doesn't overlap with Hoechst fluorescence spectrum.

The prominin-1 negative cells were eluted, and the Prominin $-1^{+}$cells were flushed at the end of the procedure after the magnetic field had been lifted. This isolation protocol is detailed in Table 2. The percentage of Prominin $-1^{+}$cells is assessed extemporaneously by flow cytometry (Figure 5A). Hundreds of thousand cells can be obtained per kidney. This yield may vary depending on experimental conditions (eg, $3.3 \pm 0.5 \times 10^{5}, 2.0 \pm 0.2 \times 10^{5}$, and $4.2 \pm 1.6 \times 10^{5}$ respectively in control, day 2 and day 9 post ischemia reperfusion injury, Additional file 5: Figure S5). Assessed by flow cytometry, the purity of Prominin-1 cells in the positive fraction was $80.0 \pm 11.1 \%$. To further verify the specificity of the enrichment in proximal tubular cells, we used real-time PCR to compare the quantity of celltype specific transcripts (using prominin-1 as a marker of proximal tubules, aquaporin 2 a marker of collecting ducts, and $C d 31$ a marker of endothelial cells). We confirmed by RT-qPCR that the enriched fraction was indeed significantly enriched in Prominin-1 mRNA compared to the eluted fraction $(\mathrm{p}=0.0304) . C d 31$ and Aqp2 mRNAs expression was very low in the enriched and the eluted fraction (Figure 5B).

\section{Cellular culture of a specific cell type isolated from a whole kidney cell suspension}

Prominin $-1^{+}$cells proved suitable for primary culture. After a period of proliferation, cells became contiguous and re-acquired an epithelial phenotype. Using contrastphase microscopy, we observed a monolayer of polygonal, contiguous cells (Figure 6A). A component of epithelial tight junctions, the transmembrane protein Zona occludens 1 (ZO-1) is a marker of differentiated epithelia. ZO-1 was found to be expressed in Prominin- $1^{+}$cells (Figure 6B). The cells expressed mRNAs of Prominin-1 and Megalin, and Ctgf, but not of Cd31 or Collagen 1 (gene expression levels differences among all group assessed by Wilcoxon test, $\mathrm{p}=0.0221$, Figure 7A). When exposed to TGF- $\beta$, Collagen 1 and Ctgf mRNAs were induced and Megalin mRNA was down-regulated ( $\mathrm{p}=0.0495$ 
Table 2 Protocol for isolation of proximal tubular cells

1. Use the cell suspension obtained in Table 1.

2. Add $20 \mu \mathrm{L}$ of FCR blocking reagent.

3. Mix well and incubate at $4^{\circ} \mathrm{C}$ for 10 minutes.

4. Add $30 \mu \mathrm{L}$ of anti-prominin-1 microbeads antibodies.

5. Mix well and incubate at $4^{\circ} \mathrm{C}$ for 10 minutes.

6. Add $10 \mu \mathrm{L}$ of anti-prominin-1 APC antibodies.

7. Mix well and incubate at $4^{\circ} \mathrm{C}$ for 5 minutes.

8. Wash cells by adding $10 \mathrm{~mL}$ of dissociating buffer.

9. Centrifuge at $500 \mathrm{~g}$ for 10 minutes.

10. Aspirate the supernatant completely.

11. Expand the cell pellet in $500 \mu \mathrm{L}$ of dissociation buffer.

12. Apply the solution to an $L S$ column primed with $3 \mathrm{~mL}$ of buffer.

13. Rinse the column with $3 \times 3 \mathrm{~mL}$ of buffer, keeping the LS column away from the magnet.

14. Collect the flow through solution and centrifuge at $500 \mathrm{~g}$ for 10 minutes.

15 Discard the supernatant and add $500 \mu \mathrm{L}$ of buffer.

16. Insert anew LS columns into an MACS separator magnet and prime it with3 $\mathrm{mL}$ of buffer.

17. Apply the cell suspension obtained in $\mathbf{1 5}$.

18. Wash the column with $3 \times 3 \mathrm{~mL}$ of buffer.

19. Remove the column from the separator and place it on a suitable collection tube.

20. Flush the magnetically labeled cells with $5 \mathrm{~mL}$ of dissociation buffer.

for each gene), whereas Prominin-1 mRNA remained stable. These phenotypic changes are typical of the effect of TGF- $\beta$ on epithelial cells, and these data suggest that Prominin-1 expression remains stable at least in the first steps of epithelial injury (Figure 7B).

\section{Discussion}

Our method can be used to analyze and separate distinct cell types from an organ (here, the kidney) during in vivo experiments. It yields operator-independent, quantitative and multi-parameter data on specific cell types. This resolves the issues of the irrelevance of cell cultures to investigate organ pathology and the cell heterogeneity characteristic of in vivo studies using whole organ lysates, which makes them unsuitable for proteomics or molecular biology.

Other approaches had been used previously: LASER micro-dissection on frozen or fixed tissues [15]; primary culture obtained by enzymatic kidney dissociation followed by FACS [16]; and, more recently, Translating Ribosome Affinity Purification (TRAP) [17].

Although these techniques can be useful, they all have some major drawbacks: 1) LASER microdissection can only be applied to dead cells (frozen or fixed, thus precluding cell culture) and cannot finely differentiate between cellular subtypes (usually, agross toluidine blue staining is performed to identify histological structures); 2) enzymatic dissociation/FACS implies a long and aggressive step of dissociation at $37^{\circ} \mathrm{C}$ when massive changes can occur within the cells $[6,18]$, biasing any subsequent analysis; in addition, cell sorters are onerous equipment which are not suitable for benchtop use; 3) TRAP is restricted to the study of translation within cells, and needs a genetically modified strain for each cell type of interest.

Our method may a have some drawbacks. First, cell dissociation does not allow to study the interactions between different kind of cells. Second, it is dependent on the quality of the antibody used to capture epithelial cells. Third, the yield may vary depending on experimental settings, but this should be considered as informative data rather than as a bias.

In addition to allowing flow cytometry analysis and cell-type specific isolation, our methods yields live cells that can be used for cell-specific primary cultures, which may be very useful for studying the phenotype of a specific cell-type in any animal strain (especially genetically modified organisms in which no cell lines are available).

\section{Conclusion}

Overall, we believe that our method offers a robust and simple tool to isolate and further study a specific cell type from an organ downstream of in vivo experimental models. This method allows a better understanding of cell-type specific biological processes in a solid organ, and provides easy cell-specific primary cultures for any chosen animal strain.

\section{Methods}

\section{Tissue dissociation}

Immediately after being harvested from adult c57bl6/J mice, each kidney was immersed in $1 \mathrm{~mL}$ of the dissociating buffer at room temperature. The renal capsule was removed and the kidney grossly chopped using a surgical blade. A cell dissociator (GentleMACS Dissociator, Miltenyl Biotec, California, USA) and dedicated tubes that preserve cell integrity (GentleMACS C-tubes, Miltenyl Biotec, California, USA) were used to dissociate cells in two sequential programs: a gentle program, followed by a stronger one, taking about 2 minutes at $4{ }^{\circ} \mathrm{C}$, with no added enzymes. Finally the supernatant was filtered through a $30-\mu \mathrm{m}$ sieve. The dissociation buffer is made with $1 \mathrm{X}$ Phosphate Buffered Serum (PBS), 0.5\% bovine serum albumin, and 2 mM EDTA.

\section{Analysis of a specific cell-type by flow cytometry, isolation of prominin- $1^{+}$cells and primary cell culture}

Flow cytometry experiments were performed using the MacsQuant 10 VBR device (Miltenyi Biotec, Bergisch Gladbach, Germany). For DNA detection, Hoechst 33342 
A

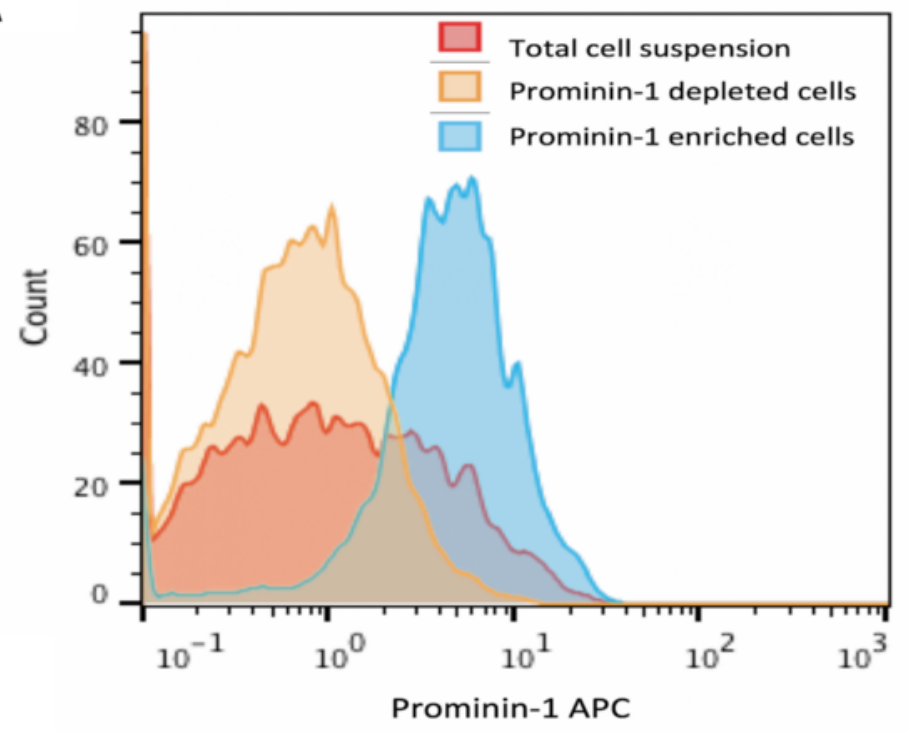

B

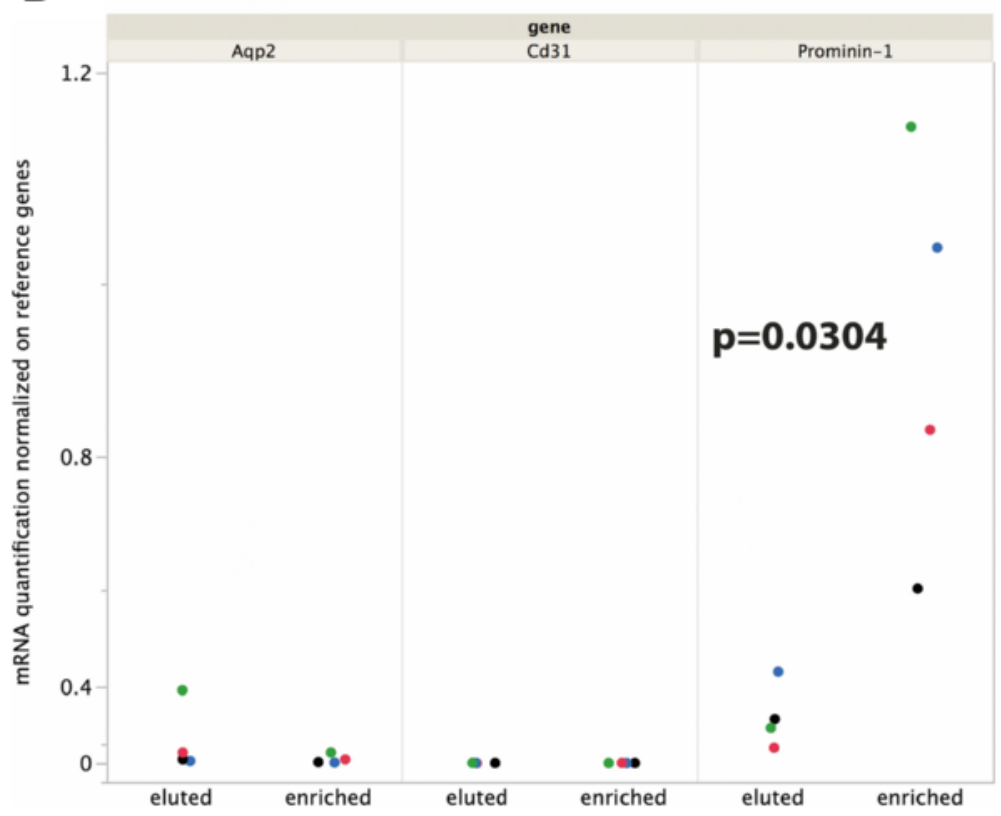

Figure 5 Specificity of proximal tubular cells enrichment. A) Density plot of prominin-1 expression showing an overlay of total kidney cell suspension in red (with a prominin-1 positive fraction estimated by flow cytometry at 37\% of total cells in the suspension), prominin-1-depleted cells in orange (with a prominin-1 positive fraction estimated by flow cytometry at $15 \%$ of total cells in the suspension), and prominin-1 enriched cells in blue (with a prominin-1 positive fraction estimated by flow cytometry at $92 \%$ of total cells in the suspension). B) RT-PCR quantification of prominin-1, Cd31 and Aap2 mRNAs in the eluted and the Prominin-1 enriched fractions. The results are shown as relative quantifications using the geometrical mean of gene Gusb and Rpl32 as the reference.

was used (Sigma Aldrich, France). The GentleMACS dissociator, GentleMACS C-tubes, anti-prominin-1 antibodies, LS columns (columns loaded with magnetic beads, Miltenyi Biotec, Bergisch Gladbach, Germany are suitable for a positive selection of magnetically labeled cells and FcR block (a solution of Fc immunoglobulin fragments designed to block non specific antibody binding by its Fc fragment to the Fc receptor expressed by some cells) were purchased from Miltenyi Biotec, Bergisch Gladbach, Germany. Full detail of the protocol is provided in Tables 1 and 2. Briefly, the cells obtained by mechanical dissociation were incubated with the proximal tubule specific anti prominin-1 antibody conjugated with magnetic microbeads. An anti APC -conjugated anti Prominin-1 

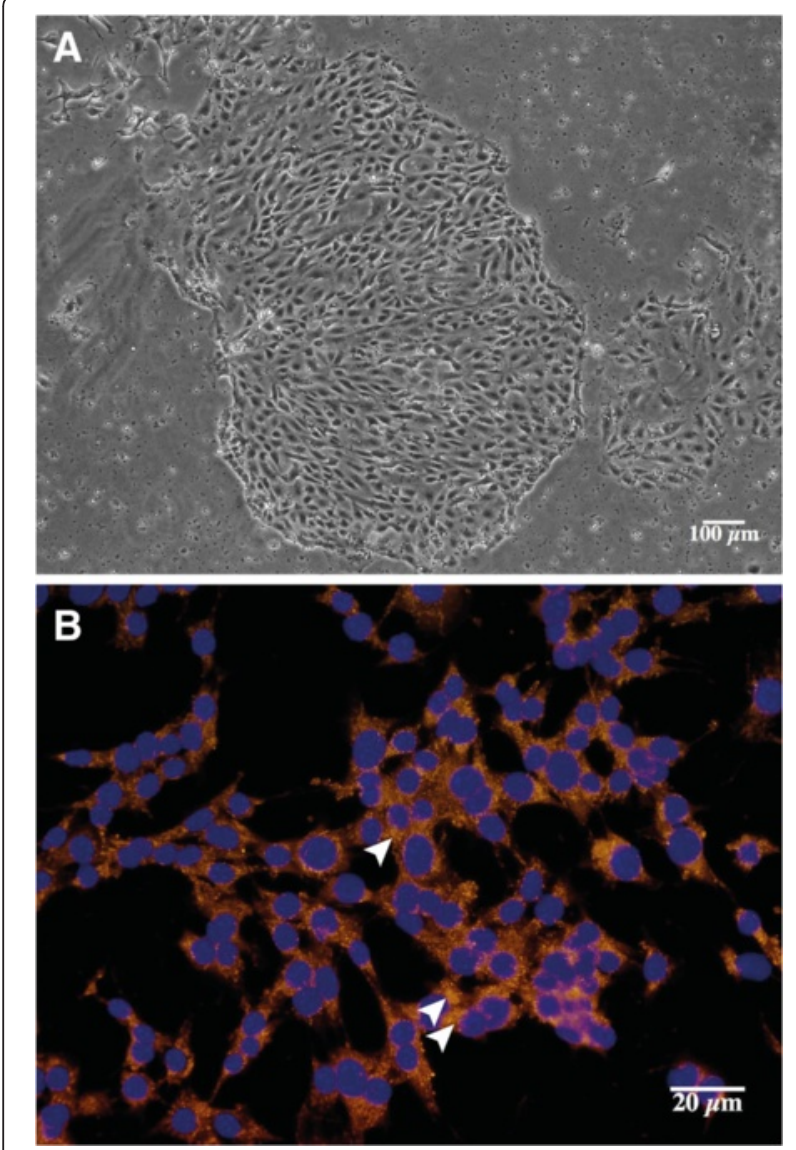

Figure 6 Primary cultures of Prominin-1+ cells. A) Contrast phase microscopy of Prominin-1+ cultured cells after 7 days. B) Immunofluorescence for ZO-1 in Prominin-1+ cultured cells. ZO-1 is expressed in the Prominin-1+ cultured cells, with a cytoplasmic pattern, enhanced at intercellular junctions (arrowheads).

antibody was then added to allow a fluorescence-based quality control of the isolation. The cells were then injected in the magnetic LS column, and rinsed in order to discard Prominin-1 negative cells in the flow-through, and to retain only Prominin $-1^{+}$cells. The LS column was then demagnetized by removing the magnet from the column, and the trapped Prominin- $1^{+}$cells were flushed in a collection tube. Cells were grown on $25 \mathrm{~cm}^{2}$ plastic flasks. The medium used for primary cell culture has been published elsewhere [19] and is detailed in the Additional file 6. For in vitro experiments, Prominin $-1^{+}$cells were stimulated with $10 \mathrm{ng} / \mathrm{mL}$ TGF- $\beta$ for 24 hours.

\section{$R N A$ extraction and RT-PCR analysis}

RNA extraction was performed with RNA isolation COLUMNS (RNeasy micro, Qiagen). RT-PCR analysis was performed on a LightCycler 480 device using the maxima first strand cDNA synthesis kit (Fermentas) for reverse transcription, SybRGreen (Fast Start DNA Master Sybr Green I; Roche Applied Science, Roche Diagnostic) and PCR primers designed with the Roche Universal Probe Library as follows: Aquaporin-2 TAGCCCTGCTCT CTCCATTG/GAGCAGCCGGTGAAATAGAT, Prominin-1 GCCCAAGCTGGAAGAATATG/CAGCAGAAAGCAGA CAATCAA, Cd31 CGGTGTTCAGCGAGATCC/CGACA GGATGGAAATCACAA, gusb CTCTGGTGGCCTTACCT GAT/CAGTTGTTGTCACCTTCACCTC, Rpl32, GCTGC CATCTGTTTTACGG/TGACTGGTGCCTGATGAACT, Ctgf, TGACCTGGAGGAAAACATTAAGA/AGCCCTGT ATGTCTTCACACTG, Collagen1 GCAGGTTCACCTAC TCTGTCCT/CTTGCCCCATTCATTTGTCT, Megalin TG GAGGATGCAGCCATATCT/GTGTGGACACTGGCACT CAG. RT-PCR was performed as follows: $95^{\circ} \mathrm{C}$ for $5 \mathrm{mi}-$ nutes, 45 cycles including $95^{\circ} \mathrm{C}$ for 15 seconds, $60^{\circ} \mathrm{C}$ for 15 seconds, and $72^{\circ} \mathrm{C}$ for 15 seconds. The melting curve was used to check the specificity of the PCR amplification (Additional file 7: Figure S6). The gene expression results were obtained by dividing the quantities of RNA for each gene by the geometrical mean of the quantity of RNA for the housekeeping genes.

\section{Immunofluorescence}

Immunofluorescence was performed on $3 \mu \mathrm{m}$ thick slides for snap frozen kidneys, and on glass slides after 15 minutes fixation at $-20^{\circ} \mathrm{C}$ with methanol for cultured cells. The slides were immersed in $1 \times$ PBS, incubated for 5 minutes in $1 \times$ PBS with $2 \%$ bovine serum albumin for blocking of non specific signal, then incubated with the primary antibody overnight at $4^{\circ} \mathrm{C}$, washed 6 times in $1 \times$ PBS, incubated for 5 minutes in $1 \times$ PBS with $2 \%$ bovine serum albumin for blocking of non specific signal, incubated with the secondary antibody for 30 minutes at room temperature in the dark and washed 6 times in $1 \times$ PBS. For negative controls, the same protocol was used without incubating with the primary antibody. Immunofluorescence study for the detection of megalin and prominin-1 was performed using a sheep anti-megalin primary antibody (1/5000) [20] with an AlexaFluor 488 anti-sheep secondary antibody (1/1000, A11055, Invitrogen, Cergy Pontoise France); and a rat anti-prominin-1 primary antibody (1/25, MB93G8 Miltenyi Biotec, Bergisch Gladbach, Germany) with an AlexaFluor 546 anti-rat secondary antibody (1/1000, A11081, Invitrogen, Cergy Pontoise France).

\section{Kidney injury models}

Mice were housed in standard conditions in the Specific Pathogen Free animal facility of INSERM, UMR-S1155: $1284 \mathrm{~L}$ ventilated boxes with a $12 / 12$ photoperiod, at a $20-$ $24^{\circ} \mathrm{C}$ temperature with a $55+/-10 \%$ humidity. Food and water were available ad libitum The kidneys were harvested from male c57bl6/J mice after or sham surgery after a renal ischemia performed by clamping of the renal artery during 30 minutes followed by reperfusion for 2,7 or 44 days. The surgery and the sacrifice were performed 

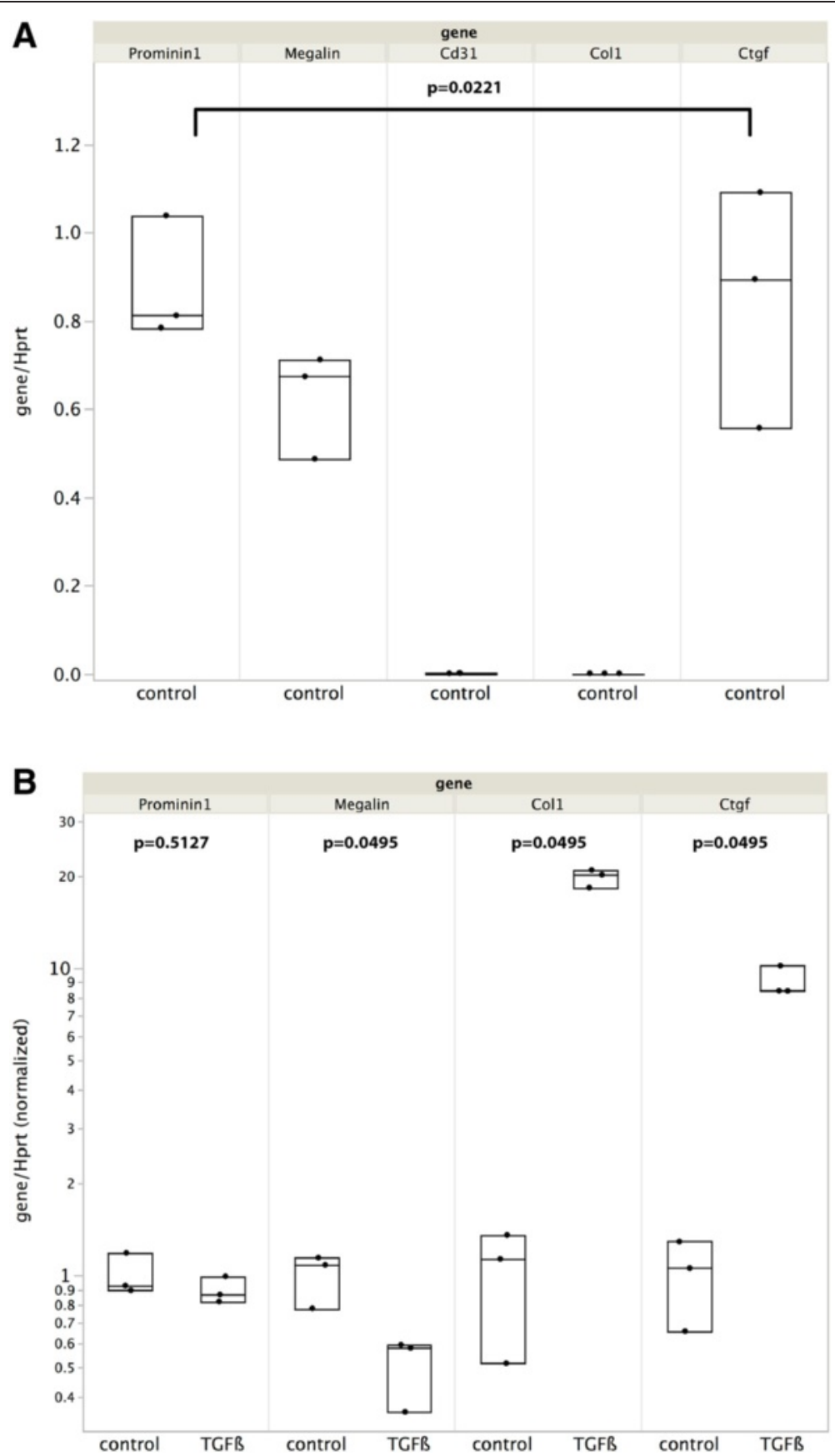

Figure 7 RT-PCR analysis of Prominin-1+ cultured cells. A) Expression of Collagen-1, Ctgf, Megalin, Cd31 and Prominin-1 mRNAs in a primary culture of Prominin-1 $1^{+}$cells. Prominin-1, Megalin and Ctgf are strongly expressed as expected in proximal tubular cells, whereas Collagen 1 and Cd31 are virtually absent. B) Effect of TGFß on Prominin-1, Megalin, Collagen-1 and Ctgf mRNAs expression. TGFß upregulated Ctgf and Collagen-1 mRNAs, downregulated Megalin mRNA, and Prominin-1 mRNA remained stable.

after anesthesia by intraperitoneal injection of penthotal. Animal care and the experimental protocol complied with the national and international guidelines and were approved by our local independent animal's ethics institution (Comité National de Réflexion Ethique sur l'Experimentation animal, numéro 5) and the French Research Ministry (authorization number 00947.02). PG has an authorization for animal experimentations ( $\left.\mathrm{N}^{\circ} \mathrm{A}-75-1934\right)$.

\section{Statistics}

Comparisons between groups were performed using a Wilcoxon test. P-values $\leq 0.05$ were considered as significant. 


\section{Additional files}

Additional file 1: Figure S1. A \& B. Gating on nucleated cells after staining with Hoechst's dye $(A)$ and the resulting study population on a Forward scatter versus Side scatter dot plot (B). C \& D. Gating on singlet cells by plotting the area under the curve by the peak of the Hoechst's signal (C) and the resulting study population on a Forward scatter versus Side scatter dot plot (D).

Additional file 2: Figure S2. A \& B: Dot plot representing Side scatter versus Megalin - FITC. Cells positive for Prominin-1 are shown in red ( negative control, B double stained sample). C \& D: Dot plot of Side scatter versus Prominin 1 - APC. Cells positive for Megalin are shown in green (C: negative control, D double stained sample). Prominin-1 and Megalin are expressed by the same cells (proximal tubular cells).

Additional file 3: Figure S3. Controls for Prominin-1 (AlexaFluor 546, red) and Megalin (AlexaFluor 488, green) immunofluorescence analysis. A: double negative control acquired through the green filter (x20). B: double negative control acquired through the red filter (x20). C: Prominin-1 single-stained control acquired through the green filter to check the absence of emission of AlexaFluor 546 through the green filter (x20). D: Megalin single stained control acquired through the red filter to check the absence of emission of AlexaFluor 488 through the red filter (x20). E \& G: Prominin-1 single-stained control (positive control) acquired through the red filter ( $E$ : x20, G: x60). F \& H: Megalin single stained control (positive control) acquired through the green filter ( $\mathrm{F}: \times 20, \mathrm{H}: \times 60)$

Additional file 4: Figure S4. Masson's Trichrome of the control (A, B) and fibrotic kidney (C, D) used for immunofluorescence in Figure 3, at magnification $\times 20(A, C)$ and $\times 60(B, D)$.

Additional file 5: Figure S5. Number of cells obtained after isolation of Prominin $-1^{+}$cells in various experimental conditions. IRI: ischemia reperfusion.

Additional file 6: Culture medium for primary culture of proximal tubular cells.

Additional file 7: Figure S6. Melting peak curves Gusb, Rpl32, Prominin-1, Megalin, Cd31, Aqp2, Collagen 1, Ctgf. No unspecific amplification was detected.

\section{Abbreviations}

RT-PCR: Reverse transcription polymerase chain reaction;

DNA: Deoxyribonucleic acid; APC: Allophycocyanin; IgG: Immunoglobulin G;

Cd31: Cluster of differentiation 31; Aqp2: Aquaporin 2; ZO-1: Zona occludens 1; LASER: Light amplification by stimulated emission of radiation;

FACS: Fluorescence activated cell sorting; TRAP: Translating ribosome affinity purification; PBS: Phosphate Buffered Serum; Gusb: Beta-glucuronidase; Rpl32: 60S ribosomal protein L32.

\section{Competing interests}

The authors declare that they have no competing interests.

\section{Authors' contributions}

$\mathrm{DL}$ carried out the magnetic isolation experiments, RT-PCR and statistics, and drafted the manuscript. $A B$ participated to the flow cytometry analysis and revised the manuscript. $\mathrm{AH}$ participated to the design of the experiments and revised the manuscript. SV carried out the cell culture and revised the manuscript. NS carried out the magnetic isolation tests and revised the manuscript. ER participated to the design of the experiments and revised the manuscript. PG designed the study, participated to the flow cytometry analyses, to the immunofluorescence study and to the interpretation of the results and completed the manuscript. All authors read and approved the final manuscript.

\section{Acknowledgements}

We thank Edith Beaugey and Perrine Frère for their help in obtaining the costaining for megalin and prominin-1, and Sandrine Placier for her help with in vitro experiments.

We thank the CORDDIM for their help in purchasing the flow cytometer used in this study. This study was supported by institutional funding from Assistance Publique - Hôpitaux de Paris and Agence de Biomédecine. PG received a scholarship from Assistance Publique -Hôpitaux de Paris, Société
Francophone de Transplantation and Société de Néphrologie. AH received an Interface INSERM-AP-HP fellowship.

\section{Author details}

${ }^{1}$ INSERM, UMR-S1155, Paris, France. ' UPMC, Sorbonne Universités, Paris 6, France. ${ }^{3}$ Urgences Néphrologiques et Transplantation Rénale, APHP, Paris, France.

Received: 23 October 2014 Accepted: 2 March 2015

Published online: 25 March 2015

\section{References}

1. Humphreys BD, Valerius MT, Kobayashi A, Mugford JW, Soeung S, Duffield JS, et al. Intrinsic epithelial cells repair the kidney after injury. Cell Stem Cell. 2008:2:284-91.

2. Hartung T, Daston G. Are in vitro tests suitable for regulatory use? Toxicol Sci. 2009;111:233-7.

3. Jenkinson SE, Chung GW, van Loon E, Bakar NS, Dalzell AM, Brown CD. The limitations of renal epithelial cell line HK-2 as a model of drug transporter expression and function in the proximal tubule. Pflugers Arch. 2012;464:601-11.

4. Lash LH. In vitro methods of assessing renal damage. Toxicol Pathol. 1998;26:33-42

5. Lash LH. Human Proximal Tubular Cells as an In Vitro Model for Drug Screening and Mechanistic Toxicology. AltTox.org. 2012,

6. Fain JN, Cheema P, Tichansky DS, Madan AK. The inflammatory response seen when human omental adipose tissue explants are incubated in primary culture is not dependent upon albumin and is primarily in the nonfat cells. J Inflamm (Lond). 2010;7:4.

7. Ruan H, Zarnowski MJ, Cushman SW, Lodish HF. Standard isolation of primary adipose cells from mouse epididymal fat pads induces inflammatory mediators and down-regulates adipocyte genes. J Biol Chem 2003;278:47585-93.

8. Seol D, Yu Y, Choe H, Jang K, Brouillette MJ, Zheng H, et al. Effect of short-term enzymatic treatment on cell migration and cartilage regeneration: in vitro organ culture of bovine articular cartilage. Tissue Eng Part A. 2014;20:1807-14.

9. Inoue T, Plieth D, Venkov CD, Xu C, Neilson EG. Antibodies against macrophages that overlap in specificity with fibroblasts. Kidney Int. 2005:67:2488-93.

10. Weigmann A, Corbeil D, Hellwig A, Huttner WB. Prominin, a novel microvillispecific polytopic membrane protein of the apical surface of epithelial cells, is targeted to plasmalemmal protrusions of non-epithelial cells. Proc Natl Acad Sci U S A. 1997;94:12425-30.

11. Bussolati B, Moggio A, Collino F, Aghemo G, D'Armento G, Grange C, et al. Hypoxia modulates the undifferentiated phenotype of human renal inner medullary CD133+ progenitors through Oct4/miR-145 balance. Am J Physiol Renal Physiol. 2012;302:F116-28.

12. Buzhor E, Omer D, Harari-Steinberg O, Dotan Z, Vax E, Pri-Chen S, et al. Reactivation of NCAM1 defines a subpopulation of human adult kidney epithelial cells with clonogenic and stem/progenitor properties. Am J Pathol. 2013;183:1621-33.

13. Vermeulen Windsant IC, Snoeijs MG, Hanssen SJ, Altintas S, Heijmans JH, Koeppel TA, et al. Hemolysis is associated with acute kidney injury during major aortic surgery. Kidney Int. 2010;77:913-20.

14. Moore JP, Sakkal S, Bullen ML, Kemp-Harper BK, Ricardo SD, Sobey CG, et al. A flow cytometric method for the analysis of macrophages in the vascular wall. J Immunol Methods. 2013;396:33-43.

15. Bonner RF, Emmert-Buck M, Cole K, Pohida T, Chuaqui R, Goldstein S, et al. Laser capture microdissection: molecular analysis of tissue. Science. 1997;278:1481,1483.

16. Van der Hauwaert C, Savary G, Gnemmi V, Glowacki F, Pottier N, Bouillez A et al. Isolation and characterization of a primary proximal tubular epithelial cell model from human kidney by CD10/CD13 double labeling. PLoS One. 2013;8:e66750.

17. Liu J, Krautzberger AM, Sui SH, Hofmann OM, Chen Y, Baetscher M, et al. Cell-specific translational profiling in acute kidney injury. J Clin Invest. 2014;124:1242-54

18. Bosio A, Huppert V, Donath S, Hennemann P, Malchow M, Heinlein UA. Isolation and enrichment of stem cells. Adv Biochem Eng Biotechnol. 2009;114:23-72. 
19. Bens M, Vallet V, Cluzeaud F, Pascual-Letallec L, Kahn A, Rafestin-Oblin ME, et al. Corticosteroid-dependent sodium transport in a novel immortalized mouse collecting duct principal cell line. J Am Soc Nephrol. 1999;10:923-34.

20. Moestrup SK, Nielsen S, Andreasen P, Jorgensen KE, Nykjaer A, Roigaard H, et al. Epithelial glycoprotein-330 mediates endocytosis of plasminogen activator-plasminogen activator inhibitor type-1 complexes. J Biol Chem. 1993;268:16564-70

Submit your next manuscript to BioMed Central and take full advantage of:

- Convenient online submission

- Thorough peer review

- No space constraints or color figure charges

- Immediate publication on acceptance

- Inclusion in PubMed, CAS, Scopus and Google Scholar

- Research which is freely available for redistribution 